\title{
ROBUSTLY NON-HYPERBOLIC TRANSITIVE ENDOMORPHISMS ON $\mathbb{T}^{2}$
}

\author{
BAOLIN HE AND SHAOBO GAN \\ (Communicated by Yingfei Yi)
}

\begin{abstract}
We prove that for any regular endomorphism $f$ on a 2 -torus $\mathbb{T}^{2}$ which is not one to one, there is a regular map $g$ homotopic to $f$ such that $g$ is $C^{1}$ robustly non-hyperbolic transitive. We also introduce interesting blender phenomena (a fat horseshoe) of 2-dimensional endomorphisms, which play an important role in our construction of some examples.
\end{abstract}

\section{INTRODUCTION}

The topic on robustly transitive diffeomorphisms attracts many people's attention. In the past several decades, people made much important progress on it. On one hand, Shub Sh2], Mañé [M1], Bonatti and Díaz [BD] gave several classes of robustly non-hyperbolic transitive diffeomorphisms respectively. However, the known robustly transitive examples are still very few. On the other hand, in DPU] and BDP it is proved that robustly transitive diffeomorphisms have some weak hyperbolicity: they have dominated splittings and their extremal bundles are volume hyperbolic. We think that they should have more dynamical consequences. For example, under some conditions their extremal bundles may be integrable. Also, there is other progress on it. For more details, readers can refer to the survey HHU.

It is well known that $C^{1}$ robustly transitive diffeomorphisms on any compact smooth surface are Anosov [M2]. However, Sumi [Su gave a $C^{1}$ robustly nonhyperbolic transitive regular endomorphism on $\mathbb{T}^{2}$ in the homotopic class of $f_{A}$, which is induced by $A=\left(\begin{array}{ll}3 & 1 \\ 1 & 1\end{array}\right)$. Let $E n d^{*}\left(\mathbb{T}^{2}\right)$ be the set of all regular endomorphisms on $\mathbb{T}^{2}\left(C^{1}\right.$ endomorphisms and determinants of their derivatives $\neq 0$ ) which are not one to one. In this paper, we construct robustly non-hyperbolic transitive regular endomorphisms of $\mathbb{T}^{2}$ in all homotopic classes of $E n d^{*}\left(\mathbb{T}^{2}\right)$.

Theorem 1.1. For any $f \in E n d^{*}\left(\mathbb{T}^{2}\right)$, there exists $g \in E n d^{*}\left(\mathbb{T}^{2}\right)$ such that $g$ is homotopic to $f$ and robustly non-hyperbolic transitive.

The homotopic classes of $E n d^{*}\left(\mathbb{T}^{2}\right)$ can be divided into two kinds: the one containing hyperbolic linear endomorphisms and the other containing non-hyperbolic linear endomorphisms. For the first kind, robustly non-hyperbolic transitive endomorphisms are derived Anosov endomorphisms $(\underline{\mathrm{Su}}]$ ). For the other kind, we

Received by the editors August 25, 2011 and, in revised form, October 23, 2011.

2010 Mathematics Subject Classification. Primary 37D30.

This work is supported by 973 program 2011CB808002 and NSFC 11025101. 
introduce a blender of 2-dimensional endomorphisms (Definition 3.1) to construct robustly non-hyperbolic transitive endomorphisms.

In $\mathrm{ABD}$, Abdenur, Bonatti and Díaz brought up the following problem:

Conjecture 1. There is a residual subset $\mathcal{R}$ of $\operatorname{Diff}^{1}(M)$ such that for any $f \in \mathcal{R}$, if the non-wandering set $\Omega(f)$ has non-empty interior, then $f$ is transitive.

The conjecture is folklore when the non-wandering set is hyperbolic. It is also proved for diffeomorphisms on any compact surface in $\mathrm{ABCD}$. But, the answer is negative for 2-dimensional endomorphisms by the following proposition, which is given in BKRU, Proposition 7.1].

Proposition 1.2. There is an open subset $\mathcal{U}$ of $E n d^{*}\left(\mathbb{T}^{2}\right)$ such that every $f \in \mathcal{U}$ has a hyperbolic attractor $\left(\neq \mathbb{T}^{2}\right)$ containing non-empty interior.

In our paper, it is a direct application of a blender of endomorphisms. The term blender (of diffeomorphisms) was first introduced by Bonatti and Díaz ([BD]) in order to construct new robustly non-hyperbolic transitive diffeomorphisms. A blender of endomorphisms also plays a similar role in our construction of examples. It is extracted from the following linear model. Let $f: C=[-1,1]^{2} \rightarrow \mathbb{R}^{2}$,

$$
f\left(x_{1}, x_{2}\right)= \begin{cases}\left(k x_{1}, \lambda x_{2}\right), & x_{1} \in\left[-\frac{1}{k}, \frac{1}{k}\right], \\ \left(k x_{1}-3, \lambda x_{2}+t\right), & x_{1} \in\left[\frac{2}{k}, \frac{4}{k}\right],\end{cases}
$$

where $t>0, \lambda \in\left(\frac{1}{2}, 1\right)$ satisfying $1-\lambda<t<\lambda$, and $k>4$. The picture of $(C, f)$ looks like a fat horseshoe. Note that $f\left(\left(\left[-\frac{1}{k}, \frac{1}{k}\right] \cup\left[\frac{2}{k}, \frac{4}{k}\right]\right) \times[-1,1]\right) \supset[-1,1] \times[0,1]$. By simple calculation, it is not difficult to show that the closure of the unstable manifold of $(0,0)$ contains the open set $(-1,1) \times(0, t)$. In fact, such property is $C^{1}$-persistent (Proposition 3.2). This model is motivated by an example in [D]. The example is as follows: $f: C \times[-1,1] \rightarrow \mathbb{R}^{2} \times \mathbb{R}$,

$$
f\left(x_{1}, x_{2}, x_{3}\right)= \begin{cases}\left(k x_{1}, \lambda x_{2}, \frac{1}{k} x_{3}\right), & x_{1} \in\left[-\frac{1}{k}, \frac{1}{k}\right], \\ \left(k x_{1}-3, \lambda x_{2}+t, \frac{1}{k} x_{3}+\frac{1}{2}\right), & x_{1} \in\left[\frac{2}{k}, \frac{4}{k}\right],\end{cases}
$$

where $t, \lambda$ and $k$ are the same as those in the above example. It is a linear model of Bonatti and Díaz's blender in $\mathrm{BD}$. Their blender has the following property: the projection of the closure of an unstable manifold of $(0,0,0)$ into the plane $\mathbb{R}^{2} \times\{0\}$ contains a non-empty interior of $\mathbb{R}^{2} \times\{0\}$ (Lemma 1.8 in $[\mathrm{BD}]$ ).

In some sense, the linear model of our blender is a special case of the endomorphisms $A_{\lambda, \tau}$ on the cylinder $\mathbb{R} / \mathbb{Z} \times \mathbb{R}: \quad A_{\lambda, \tau}(\theta, t)=(k \theta, \lambda t+\tau(\theta))$, where $k$ is a positive integer, $\lambda \in(0,1)$ and $\tau$ is a continuous function. Tsujii [T] first considered these from a probabilistic perspective. Later, Bamón, Kiwi, RiveraLetelier and Urzúa [BKRU] also studied their topological properties. Also, these endomorphisms on a cylinder can be regarded as a generalization of fat baker's transformations ( $\mathrm{AY}]$ ).

Here, we give some description on the construction of robustly non-hyperbolic transitive endomorphisms in the homotopic classes of $E n d^{*}\left(\mathbb{T}^{2}\right)$, which contains non-hyperbolic linear endomorphisms. We $\left(C^{1}\right)$ perturb a non-hyperbolic linear endomorphism to get a blender and, at the same time, control the perturbed dynamics with some stable global properties to make it robustly transitive (Example 3.6). This is just the principle of constructing robustly non-hyperbolic transitive diffeomorphisms in $[\mathrm{BD}$. 
In the end, we make some comparisons between regular endomorphisms and diffeomorphisms. Of course, some local arguments of diffeomorphisms, e.g., $\lambda$ lemma and the shadowing lemma of hyperbolic sets $([\mathrm{L})$, are still valid for regular endomorphisms. But hyperbolic sets are generally not stable (see Remark 3.5). Also, the normal hyperbolic theory is not always valid for endomorphisms. For example, the set $S^{1} \times\{0\}$ of $f_{0}$ in the proof of Proposition 1.2 is normally contracting and a hyperbolic attractor, but a small perturbation of $f_{0}$ may have an attractor with non-empty interior. But in the normally expanding case, Berger Be] proved some positive results.

It is natural to ask "do robustly transitive endomorphisms have any weak hyperbolicity?" Since they can have sources, we cannot expect them to have dominated splitting. Fortunately, the ergodic closing lemma ( $[\mathrm{Mo})$ is still valid for regular endomorphisms. Then it is not difficult to show that "they (not one to one) are volume expanding." For details, the reader can refer to [LP], which also gives a sufficient condition for a transitive endomorphism to be robustly transitive.

\section{Definitions AND NOtATion}

In this paper, manifolds are $C^{\infty}$ smooth compact Riemannian manifolds without boundary, and maps are $C^{1}$ smooth maps.

Let $M$ be a compact Riemannian manifold, $\|$.$\| be a Riemannian metric on$ $M$, and $\operatorname{End}(M)$ be the set of all $\left(C^{1}\right)$ regular endomorphisms on $M$ equipped with the usual $C^{1}$ topology. Note that $\operatorname{End}(M)$ contains diffeomorphisms. Given $f \in \operatorname{End}(M)$, denote by $D f$ the derivative operator of $f$. Since the determinant of $D f$ is not equal to zero, $f$ is a covering map.

Let $E$ and $F$ be two sub-bundles of $T M$ satisfying $E \oplus F=T M$. Define two cone fields $\mathcal{C}_{\alpha}^{E}$ and $\mathcal{C}_{\alpha}^{F}$ on $T M$ :

$$
\begin{aligned}
& \mathcal{C}_{\alpha}^{E}(z)=\left\{v \in T_{z} M \mid v=\left(v^{E}, v^{F}\right),\left\|v^{F}\right\|<\alpha\left\|v^{E}\right\|\right\}, \\
& \mathcal{C}_{\alpha}^{F}(z)=\left\{v \in T_{z} M \mid v=\left(v^{E}, v^{F}\right),\left\|v^{E}\right\|<\alpha\left\|v^{F}\right\|\right\} .
\end{aligned}
$$

Definition 2.1. An invariant subset $\Lambda(f(\Lambda)=\Lambda)$ of $f$ is said to be hyperbolic if $T \Lambda$ has the bundle splitting $T_{\Lambda} M=E \oplus F$ and there is $\alpha>\alpha^{\prime}>0$ and $\lambda>1$ such that, for any $x \in \Lambda$ and any unit vector $v \in \mathcal{C}_{\alpha}^{F}(x)$,

$$
\operatorname{Df}\left(\mathcal{C}_{\alpha}^{F}(x)\right) \subset \mathcal{C}_{\alpha^{\prime}}^{F}(f(x)), \quad\|D f(v)\|>\lambda,
$$

and such that for any $x \in \Lambda$, any unit vector $v \in \mathcal{C}_{\alpha}^{E}(f(x))$, and the diffeomorphism $g$ on a neighborhood of $f(x)$ satisfying that $g f$ is the identity on a neighborhood of $x$,

$$
D g\left(\mathcal{C}_{\alpha}^{E}(f(x))\right) \subset \mathcal{C}_{\alpha^{\prime}}^{E}(x), \quad\|D g(v)\|>\lambda .
$$

We say that $f$ is Anosov if $f$ is hyperbolic on $M$. Also, $f$ is said to be an expanding map if it is an Anosov map without contracting direction $(E=0)$.

Suppose $p$ is a hyperbolic fixed point of $f$. Then there is a neighborhood $V$ of $p$ and a neighborhood $\mathcal{U}$ of $f$ in $\operatorname{End}(M)$ such that for any $g \in \mathcal{U}, g$ has the unique hyperbolic fixed point $p_{g}$ in $V$ (the continuation of $\left.p_{f}=p\right), p_{g}$ has a local $C^{1}$ stable (unstable) manifold $W_{l o c}^{s}\left(p_{g}, g\right)\left(W_{l o c}^{u}\left(p_{g}, g\right)\right)$ of a uniform size, and the local stable 
and unstable manifolds of $p_{g}$ continuously vary with $g$. Let the stable manifold of $p$ be

$$
W^{s}(p, f)=\bigcup_{n=0}^{+\infty} f^{-n}\left(W_{l o c}^{s}(p, f)\right) \quad\left(=\left\{x \in M \mid \lim _{n \rightarrow \infty} d\left(f^{n}(x), p\right)=0\right\}\right)
$$

and the unstable manifold of $p$ be

$$
W^{u}(p, f)=\bigcup_{n=0}^{+\infty} f^{n}\left(W_{l o c}^{u}(p, f)\right) .
$$

Remark 2.2. Generally, the stable and unstable manifolds are not injective submanifolds. The definition of hyperbolicity is equivalent to the one in $\left[\mathrm{P}\right.$. Let $\left\{x_{n}\right\}_{n=-\infty}^{+\infty}$ be an orbit of $f$. The $D f$-invariant expanding space

$$
E^{u}\left(\left\{x_{n}\right\}\right)=\bigcap_{n=0}^{\infty} D f^{n}\left(\mathcal{C}_{\alpha}^{F}\left(x_{-n}\right)\right)
$$

depends upon the choice of negative orbits. So, Przytychi $[\mathrm{P}$ considered local unstable manifolds of orbits, which have similar properties with those in the classic hyperbolic theory of diffeomorphisms. The unstable manifold of a hyperbolic fixed point $p$ defined here is just the unstable manifold of the orbit $(\ldots, p, p, \ldots)$ in $[\mathrm{P}$.

A compact invariant set $\Lambda$ of $f$ is said to be transitive if there is a positive orbit $\left\{f^{n} x \mid n \geq 0\right\}$ dense in $\Lambda$. It is equivalent to one of the following conditions: (i) for any non-empty open subset $U$ of $\Lambda, \overline{\bigcup_{n=0}^{\infty} f^{n}(U)}=\Lambda$; (ii) for any non-empty open subset $U$ of $\Lambda, \overline{\bigcup_{n=0}^{\infty} f^{-n}(U)}=\Lambda$.

A compact invariant set $\Lambda$ is said to be a hyperbolic attractor if $\Lambda \neq M$ is hyperbolic and transitive and if there is an open subset $U$ of $M$ satisfying $f(\bar{U}) \subset U$ and $\bigcap_{n=0}^{\infty} f^{n}(\bar{U})=\Lambda$. The corresponding open set $U$ is said to be a trapping region of the attractor $\Lambda$.

We say that $f$ is robustly non-hyperbolic transitive if there is a neighborhood $\mathcal{U}(f)$ of $f$ in $\operatorname{End}(M)$ such that every $g \in \mathcal{U}(f)$ is transitive and not Anosov.

For a matrix $A$, denote all eigenvalues of $A$ by $\sigma(A)$. A map $f: \mathbb{R}^{2} \rightarrow \mathbb{R}^{2}$ is said to be a $\tau$-periodic map if $f(x+v)=f(x)$ for any $x \in \mathbb{R}^{2}, v=(i \tau, j \tau),(i, j) \in \mathbb{Z}^{2}$. Let $P$ be a 1 -periodic map on $\mathbb{R}^{2}$ and $A \in G L(2, \mathbb{Z})$. Then $F=A+P$ induces a unique endomorphism $f$ on $\mathbb{T}^{2}$ satisfying $\pi F=f \pi$, where $\pi$ is the standard covering map from $\mathbb{R}^{2}$ to $\mathbb{T}^{2}$. The endomorphism on $\mathbb{T}^{2}$ induced by a linear map $A \in G L(2, \mathbb{Z})$ is denoted by $f_{A}$. For any endomorphism $f$ on $\mathbb{T}^{2}$, there is $A \in G L(2, \mathbb{Z})$ and a lifting $F=A+P$ ( $P$ is 1-periodic) satisfying $\pi F=f \pi$ (see $[\mathrm{AP}$ ). Note that $f$ is homotopic to $f_{A}$ in the space of all continuous endomorphisms on $\mathbb{T}^{2}$. Then according to the linear representative $f_{A}$, we divide the homotopic classes of $E n d^{*}\left(\mathbb{T}^{2}\right)$ into three types:

(i) $\sigma(A)=\left\{\lambda_{1}, \lambda_{2}\right\},\left|\lambda_{1}\right|<1<\left|\lambda_{2}\right|$.

(ii) $\sigma(A)=\left\{\lambda_{1}, \lambda_{2}\right\},\left|\lambda_{1}\right|=1,\left|\lambda_{2}\right|=k>1$.

(iii) $\sigma(A)=\left\{\lambda_{1}, \lambda_{2}\right\}, 1<\left|\lambda_{1}\right| \leq\left|\lambda_{2}\right|$.

The robustly non-hyperbolic transitive regular endomorphisms on $\mathbb{T}^{2}$ given in $\mathrm{Su}$ belong to the first type. Following his construction and proof, we can get the same conclusion for all homotopic classes of $E n d^{*}\left(\mathbb{T}^{2}\right)$ of type (i). To prove Theorem 1.1, we will need to deal only with type (ii) (Proposition 3.7) and type (iii) (Proposition 4.1). 


\section{A BLENDER OF ENDOMORPHISMS}

In this section, we give the definition of a blender of endomorphisms and use blender phenomena to construct two examples on $\mathbb{T}^{2}$ : (1) robustly hyperbolic attractors with non-empty interior; (2) robustly non-hyperbolic transitive regular endomorphisms of type (ii).

Let $N^{u}, N^{c s}$ be two smooth Riemannian manifolds, $M=N^{u} \times N^{c s}$. Then $T M=T\left(N^{u}\right) \oplus T\left(N^{c s}\right)$. Also, let $\mathcal{C}_{\alpha}^{u}$ and $\mathcal{C}_{\alpha}^{c s}$ be two cone fields around the subbundles $T\left(N^{u}\right)$ and $T\left(N^{c s}\right)$ respectively. A $\mathcal{C}_{\alpha}^{u}\left(\mathcal{C}_{\alpha}^{c s}\right)$ curve $\gamma$ is a smooth curve on $M$ satisfying the tangent space $T \gamma \subset \mathcal{C}_{\alpha}^{u}\left(T \gamma \subset \mathcal{C}_{\alpha}^{c s}\right)$. In this section, $N^{u}=N^{c s}$ will be an interval of $\mathbb{R}$ or $S^{1}$.

Let $C=[-1,1]^{u} \times[-1,1]^{c s}, u=c s=1$. A smooth $\mathcal{C}_{\alpha}^{u}$ curve $\gamma \subset[-1,1] \times(-1,1)$ is said to be a u-curve of $C$ if $\gamma \cap\{ \pm 1\} \times[-1,1]=\partial \gamma$.

Definition 3.1. Let $f: C \rightarrow \mathbb{R}^{2}$ be a $C^{1}$ map. $(C, f)$ is said to be a blender if there exist two compact subsets $A_{1}$ and $A_{2}$ of $(-1,1)^{2}$ such that:

1) $\left.f\right|_{A_{i}}$ is a diffeomorphism onto its image, which is denoted by $f_{i}, i=1,2$.

2) $f$ is hyperbolic on $A_{1} \cup A_{2}$ (the cone fields $\mathcal{C}^{u}$ and $\mathcal{C}^{c s}$ are defined in $\mathbb{R}^{2}$ ). More precisely, there is $0<\alpha^{\prime}<\alpha$ and $\lambda>1$ such that, for any $x \in f_{i}\left(A_{i}\right)$ and any unit vector $v \in \mathcal{C}_{\alpha}^{c s}(x)$, one has

$$
D f_{i}^{-1}\left(\mathcal{C}_{\alpha}^{c s}(x)\right) \subset \mathcal{C}_{\alpha^{\prime}}^{c s}\left(f_{i}^{-1} x\right), \quad\left\|D f_{i}^{-1}(v)\right\|>\lambda, \quad i=1,2,
$$

and for any $x \in A_{1} \cup A_{2}$ and any unit vector $v \in \mathcal{C}_{\alpha}^{c s}(x)$, one has

$$
D f\left(\mathcal{C}_{\alpha}^{u}(x)\right) \subset \mathcal{C}_{\alpha^{\prime}}^{u}(f x), \quad\|D f(v)\|>\lambda .
$$

3) There are two $u$-curves $\gamma_{1}$ and $\gamma_{2}$ of $C$ such that $f\left(\gamma_{1} \cap A_{i}\right) \supset \gamma_{i}, i=1,2$, and $\gamma_{2}$ is strictly above $\gamma_{1}$.

4) Let $V_{1}$ be the closed subset of $C$ between $\gamma_{1}$ and $\gamma_{2}$, and let $V_{2}$ be the closed subset of $C$ not below the curve $\gamma_{2}$. Also, let $A_{i}^{\prime}$ be the closed subset of $A_{i}$ not below the curve $\gamma_{1}, i=1,2 . f\left(A_{i}^{\prime}\right)$ contains $V_{i}, i=1,2$.

Now we check that the $C^{1}$ map $f:[-1,1]^{2} \rightarrow \mathbb{R}^{2}$ in the introduction is a blender. Take $0<a<1$ satisfying that $1-\lambda a<t<\lambda a$. Let $A_{1}=\left[-\frac{1}{k}, \frac{1}{k}\right] \times[-a, a]$ and $A_{2}=\left[\frac{2}{k}, \frac{4}{k}\right] \times[-a, a]$. It is not difficult to show that $\left.f\right|_{A_{1} \cup A_{2}}$ satisfies all conditions of a blender.

Note that $f\left(\gamma_{1} \cap A_{1}\right) \supset \gamma_{1}$ and $f$ uniformly expands on the curve $f_{1}^{-1}\left(\gamma_{1}\right)\left(\subset \gamma_{1}\right)$. Then $f$ has a hyperbolic fixed saddle $p \in \gamma_{1}$ and $\gamma_{1} \cup \gamma_{2}$ is contained in some positive iteration of $f$ on a local unstable manifold of $p$. For convenience, $\gamma_{1}$ is also said to be a local unstable manifold of $p$. Now we show two properties of a blender: the closure of the unstable manifold of $p$ contains a non-empty interior, and a blender phenomenon is $C^{1}$ persistent.

Proposition 3.2. If $(C, f)$ is a blender, then:

(1) $\overline{W^{u}(p, f)}$ contains the subset of $C$ above the local unstable manifold $\gamma_{1}$ of $p$

(2) there exists a neighborhood $\mathcal{U}(f)$ of $f$ in $C^{1}\left(C, \mathbb{R}^{2}\right)$ such that for any $g \in$ $\mathcal{U}(f),(C, g)$ is a blender.

Proof. (1) Suppose on the contrary that there exists a $\mathcal{C}_{\alpha}^{c s}$ curve $\gamma^{0}$ in the region $V_{1} \cup V_{2}$ such that $\gamma^{0} \cap \overline{W^{u}(p, f)}=\emptyset$. Then $f^{-n}\left(\gamma^{0}\right) \cap \gamma_{2}=\emptyset$ for any integer $n \geq 0$. Suppose $\gamma^{0} \subset V_{i}\left(\subset f\left(A_{i}^{\prime}\right)\right), i=1$ or 2 . Then the $\mathcal{C}_{\alpha}^{c s}$ curve 
$\gamma^{1}=f_{i}^{-1}\left(\gamma^{0}\right) \subset A_{i}^{\prime} \subset V_{1} \cup V_{2}$ and $\ell\left(\gamma^{1}\right)>\lambda \ell\left(\gamma^{0}\right)$. Inductively, $f^{-n}\left(\gamma^{0}\right)$ contains a $\mathcal{C}_{\alpha}^{c s}$ curve $\gamma^{n}$ for any integer $n>0$, and the length of the curve $\gamma^{n}$ increases to infinity as $n \rightarrow+\infty$. A contradiction!

(2) Note that $\gamma_{1} \cup \gamma_{2}$ intersects transversely with $\{ \pm 1\} \times[-1,1]$ and $\gamma_{1} \cup \gamma_{2}$ is contained in the finite-time iteration of $f$ on the local unstable manifold of the hyperbolic fixed point $p$. Then there exist two closed neighborhoods $B_{1}$ and $B_{2}$ of $A_{1}$ and $A_{2}$, respectively, and a neighborhood $\mathcal{U}$ of $f$ such that for any $g \in \mathcal{U}$, one has that

1) $\left.g\right|_{B_{1} \cup B_{2}}$ satisfies conditions 1),2) and 3) of a blender. Let $\sigma(g)$ be the continuation of $\sigma, \sigma=\gamma_{1}, \gamma_{2}, V_{1}, V_{2}$, and $A_{i}(g)=B_{i}, i=1,2$. Also, the definition of $A_{i}^{\prime}(g)$ and $g_{i}$ is parallel with $A_{i}^{\prime}$ and $f_{i}$ respectively, $i=1,2$.

2) $g\left(A_{i}(g)\right) \supset V_{i}(g)$ and $g\left(A_{i}^{\prime}(g)\right)$ intersects with the interior of $V_{i}(g)$.

Note that for any $g \in \mathcal{U}, g_{i}^{-1}\left(g\left(A_{i}(g)\right) \cap\{ \pm 1\} \times[-1,1]\right)$ consists of two disjoint $\mathcal{C}_{\alpha}^{c s}$ curves, $i=1,2$. Combining this with $g_{i}^{-1}\left(\gamma_{i}(g)\right) \subset \gamma_{1}(g)$, $g\left(A_{i}(g)\right) \supset V_{i}(g)$ and $g\left(A_{i}^{\prime}(g)\right)$ intersects with the interior of $V_{i}(g)$, one has that $A_{i}^{\prime}(g)$ contains $\partial\left(g_{i}^{-1}\left(V_{i}(g)\right)\right), i=1,2$. Therefore, $g\left(A_{i}^{\prime}(g)\right) \supset V_{i}(g), i=$ 1,2 .

Remark 3.3. The blender phenomenon only relies on the map $\left.f\right|_{A_{1} \cup A_{2}}$, and the hyperbolicity on $\mathcal{C}_{\alpha}^{u}\left(\left.D f\right|_{A_{1} \cup A_{2}}\right.$ preserves $C_{\alpha}^{u}$ and uniformly expands over $\left.C_{\alpha}^{u}\right)$ is not necessary. We only demand that $\gamma_{1}$ is a finite-time iteration of $f$ on a local unstable manifold of $p$ and that $\gamma_{1} \cup \gamma_{2}$ intersects transversely with $\{ \pm 1\} \times[-1,1]$ exactly in $\partial \gamma_{1} \cup \partial \gamma_{2}$.

In this section, let $\mathbb{T}^{2}=\left[-\frac{1}{2}, \frac{1}{2}\right]^{2} / \mathbb{Z}^{2}=S^{1} \times S^{1}$. Here, we give an example of robustly hyperbolic attractors as an application of a blender. For the reader's convenience, we repeat its statement.

Proposition 1.2. There is an open subset $\mathcal{U}$ of $\operatorname{End}^{*}\left(\mathbb{T}^{2}\right)$ such that every $f \in \mathcal{U}$ has a hyperbolic attractor $\left(\neq \mathbb{T}^{2}\right)$ containing non-empty interior.

To prove the proposition, we need a lemma on transitivity.

Lemma 3.4. Suppose $\Lambda$ is a hyperbolic attractor of a regular endomorphism $f$ on a manifold $M$ and $U$ is a trapping region of $\Lambda$. Then there is a neighborhood $\mathcal{U}$ of $f$ such that for any $g \in \mathcal{U}, g$ has a hyperbolic attractor $\Lambda_{g}=\bigcap_{n=0}^{+\infty} g^{n}(U)$.

This can be deduced by the following facts:

1) three properties of hyperbolic orbit spaces: expansivity (Proposition 2.4 in $[\mathrm{L}]$ ), shadowing property (Proposition 2.6 in $[\mathrm{L}]$ ) and $\varepsilon$-stability of hyperbolic orbit spaces (Theorem $2.7 \mathrm{a}$ in $[\mathrm{L}]$ );

2) the local maximality of attractors;

3) for any compact invariant subset $\Delta$ of a regular endomorphism $g$ on a manifold $M,(\Delta, g)$ is transitive iff $\left(\widetilde{\Delta}, \sigma_{g}\right)$ is transitive.

Here, the orbit space (inverse limit space) $\widetilde{\Delta}$ of an invariant subset $\Delta$ of $g$ is the set $\left\{\tilde{x}=\left\{x_{i}\right\}_{-\infty}^{+\infty} \mid x_{i} \in \Delta, g\left(x_{i}\right)=x_{i+1}, i \in \mathbb{Z}\right\}$. Also, $\left.g\right|_{\Delta}$ induces the left shift operator $\sigma_{g}: \widetilde{\Delta} \rightarrow \widetilde{\Delta}$ which satisfies $\pi \sigma_{g}=g \pi$, where $\pi$ is the projection to its zeroth coordinate. Since the proof of the lemma is basic, we only give a sketch of it. The proof of the last fact is trivial. By the first two facts, we can deduce that there is a neighborhood $\mathcal{U}$ of $f$ such that for any $g \in \mathcal{U},\left(\widetilde{\Lambda_{g}}, \sigma_{g}\right)$ is topologically 
conjugate to $\left(\widetilde{\Lambda_{f}}, \sigma_{f}\right)$. Its proof parallels that of diffeomorphisms. By the last fact, we see that $\left(\Lambda_{g}, g\right)$ is an attractor.

Proof of Proposition 1.2. The construction is simple: just take a hyperbolic attractor with a blender. Let $k>5$ be an integer, $I_{1}=\left[-\frac{1}{4 k}, \frac{1}{4 k}\right], I_{2}=\left[\frac{3}{4 k}, \frac{5}{4 k}\right], t>0$ and $\frac{1}{2}<\lambda<1$ satisfying $t<\frac{1-\lambda}{3}$. Also, take a smooth 1-periodic function $\alpha$ satisfying that $\alpha\left(I_{1}\right)=0, \alpha\left(I_{2}\right)=1$ and $0 \leq \alpha \leq 1$. Construct regular endomorphisms $f_{t}$ on $\mathbb{T}^{2}$ :

$$
f_{t}\left(x_{1}, x_{2}\right)=\left(k x_{1}, \lambda x_{2}+\alpha\left(x_{1}\right) t\right), \quad\left(x_{1}, x_{2}\right) \in S^{1} \times\left[-\frac{1}{3}, \frac{1}{3}\right] .
$$

Since $0 \leq \alpha \leq 1$ and $0<t<\frac{1-\lambda}{3}, f_{t}\left(S^{1} \times\left[-\frac{1}{3}, \frac{1}{3}\right]\right) \subset S^{1} \times\left[-\frac{\lambda}{3}, \frac{\lambda}{3}+t\right] \subset S^{1} \times\left(-\frac{1}{3}, \frac{1}{3}\right)$. Additionally, note that $\Lambda_{f_{0}}=\bigcap_{n=0}^{\infty} f_{0}{ }^{n}\left(S^{1} \times\left[-\frac{1}{3}, \frac{1}{3}\right]\right)$ is a hyperbolic attractor of $f_{0}$. So, by the above lemma, there is a neighborhood $\mathcal{U}_{1}$ of $f_{0}$ such that for any $g \in \mathcal{U}_{1}$, the compact invariant set $\Lambda_{g}=\bigcap_{n=0}^{\infty} g^{n}\left(S^{1} \times\left[-\frac{1}{3}, \frac{1}{3}\right]\right)$ is a hyperbolic attractor. Suppose $f=f_{t} \in \mathcal{U}_{1}$, and let $p_{f}=(0,0)$. Take $a>0$ satisfying $a-\lambda a<t<\lambda a$. Let $C=\left[-\frac{1}{4}, \frac{1}{4}\right] \times[-a, a]$ and $A_{i}=I_{i} \times[-a, a], i=1,2$. We regard $\left.f\right|_{A_{1} \cup A_{2}}$ as a map from $A_{1} \cup A_{2}$ to $\left[-\frac{1}{2}, \frac{1}{2}\right]^{2}$ :

$$
f\left(x_{1}, x_{2}\right)= \begin{cases}\left(k x_{1}, \lambda x_{2}\right), & \left(x_{1}, x_{2}\right) \in A_{1}, \\ \left(k x_{1}-1, \lambda x_{2}+t\right), & \left(x_{1}, x_{2}\right) \in A_{2} .\end{cases}
$$

So $(C, f)$ is a blender. Consequently, by the properties of a blender, there exists a neighborhood $\mathcal{U} \subset \mathcal{U}_{1}$ of $f$ such that for any $g \in \mathcal{U}$, the hyperbolic attractor $\Lambda_{g}$ $\supset \overline{W^{u}\left(p_{g}, g\right)}\left(g\left(W^{u}\left(p_{g}, g\right)\right)=W^{u}\left(p_{g}, g\right)\right)$ contains a non-empty interior.

Remark 3.5. Note that the hyperbolic attractor $\Lambda_{f_{0}}$ is not (structurally) stable. Generally, hyperbolic sets of endomorphisms are not stable. In [P], Przytycki pointed out the astonishing fact that "for any non-expanding and non-injective Anosov endomorphism, there are uncountable Anosov endomorphisms contained in any neighborhood of the Anosov endomorphism such that any two of them are not topologically conjugated." The complexity of Anosov endomorphisms is mainly caused by the fact that the expanding direction relies on negative orbits. In [AH], Aoki and Hiraide classified some special Anosov endomorphisms on $\mathbb{T}^{n}$ by topological conjugation.

Now we construct robustly non-hyperbolic transitive examples by the phenomenon of a blender. For the convenience of the reader, we first give a sketch of the construction in the homotopic class of $f_{A}, A=\operatorname{diag}(k, 1), k>5$.

Example 3.6. For simplicity, in this example we identify $\mathbb{T}^{2}=\mathbb{R}^{2} /[-1,1]^{2}$. But we still use the identification $\mathbb{T}^{2}=\mathbb{R}^{2} /[-1 / 2,1 / 2]^{2}$ elsewhere.

Let $t, a, \lambda$ satisfy $0<1-\lambda \ll 1-a \ll t \ll 1$, and $\varepsilon=\frac{1}{100 k}$. Also, let $A_{0}=$ $\left[-\frac{1}{2 k}-\varepsilon, \frac{1}{2 k}+\varepsilon\right] \times[-a, a], A_{6}=\left[\frac{3}{2 k}-\varepsilon, \frac{5}{2 k}+\varepsilon\right] \times[-a, a]$, and $B_{6}=\left[\frac{3}{2 k}-\varepsilon, \frac{5}{2 k}+\varepsilon\right] \times S^{1}$, $C=\left[-\frac{1}{2}, \frac{1}{2}\right] \times[-a, a]$. Take a $C^{1}$-perturbation $f$ of $f_{A}$ satisfying the following conditions:

i) $f\left(x_{1}, x_{2}\right)= \begin{cases}\left(k x_{1}, \lambda x_{2}\right), & \left(x_{1}, x_{2}\right) \in A_{0}, \\ \left(k x_{1}-2, \lambda x_{2}+t\right), & \left(x_{1}, x_{2}\right) \in A_{6},\end{cases}$

ii) for fixed $t$ and $a,\left.f\right|_{B_{6}}$ is $C^{1}$-close to the map $\left.\left(f_{A}+t\right)\right|_{B_{6}}$.

Here, we only give an explanation as to why $f$ is robustly transitive. Since $(C, f)$ is a blender, $\overline{W^{u}(p, f)}(\mathrm{p}=(0,0))$ contains $\left[-\frac{1}{2}, \frac{1}{2}\right] \times\left[\frac{a}{2}, a\right]$. Note that $\left.f\right|_{B_{6}}$ is nearly a 
rotation $R_{t}$ in the direction $(0,1)$ for fixed $t$ and $a$, and $f$ is $C^{1}$ close to $f_{A}$. Then we deduce that $\overline{W^{u}(p, f)}(\mathrm{p}=(0,0))$ contains $\left[-\frac{1}{2}, \frac{1}{2}\right] \times S^{1}$. By the uniform expansion on $C_{\alpha}^{u}$ and the $D f$-invariance of $C_{\alpha}^{u}$, we can see that $\overline{W^{u}(p, f)}=\mathbb{T}^{2}$. For any $C_{\alpha}^{u}$ curve $\gamma$, by the uniform expansion on $C_{\alpha}^{u}$ and the $D f$-invariance of $C_{\alpha}^{u}$ again, we see that there is a positive integer $m$ such that $f^{m}(\gamma)$ runs through $B_{6}$ in the direction $(1,0)$. Again, note that $\left.f\right|_{B_{6}}$ is nearly a rotation $R_{t}$ in the direction $(0,1)$ for fixed $t$ and $a$. We can see that $\bigcup_{n=0}^{\infty} f^{n}(\gamma) \cap W_{l o c}^{c s}(p, f) \neq \varnothing$. So, $W^{s}(p, f)$ is dense in $\mathbb{T}^{2}$. Furthermore, by the $\lambda$-lemma, we get the transitivity of $f$. Note that the properties in our analysis are all robust. Therefore, $f$ is robustly transitive.

Proposition 3.7. Suppose $A \in G L(2, \mathbb{Z}), \sigma(A)=\left\{\lambda_{1}, \lambda_{2}\right\},\left|\lambda_{1}\right|=k>1,\left|\lambda_{2}\right|=1$. Then there exists an open set $\mathcal{U}$ of $E n d^{*}\left(\mathbb{T}^{2}\right)$ such that $f_{A} \in \overline{\mathcal{U}}$ and every $g \in \mathcal{U}$ is transitive and not Anosov.

Proof. Let $\alpha=(a, b)$ satisfy $\alpha A=\lambda_{1} \alpha$, where $a$ and $b$ are two integers and coprime. We can take another integer vector $\beta$ such that $\left(\begin{array}{c}\alpha \\ \beta\end{array}\right) \in S L(2, \mathbb{Z})$. Let $P=\left(\begin{array}{c}\alpha \\ \beta\end{array}\right)$. Then $\left(\begin{array}{c}\alpha \\ \beta\end{array}\right) A=\left(\begin{array}{c}\lambda_{1} \alpha \\ t_{1} \alpha+t_{2} \beta\end{array}\right)$, where $t_{1}$ and $t_{2}$ are two rational numbers, i.e., $P A P^{-1}=\left(\begin{array}{cc}\lambda_{1} & 0 \\ t_{1} & t_{2}\end{array}\right)$. Note that $t_{2}=\lambda_{2}$ and $t_{1}$ is an integer. Without loss of generality, let $A=\left(\begin{array}{cc}\lambda_{1} & 0 \\ t_{1} & \lambda_{2}\end{array}\right)$. Take $Q=\left(\begin{array}{cc}\lambda_{1}-\lambda_{2} & 0 \\ t_{1} & 1\end{array}\right)$, $B=\left(\begin{array}{cc}\lambda_{1} & 0 \\ 0 & \lambda_{2}\end{array}\right)$; then $A Q=Q B$. Also, let $\tau=\frac{1}{\left|\lambda_{1}-\lambda_{2}\right|}$.

Claim 3.8. There exists $F \in \operatorname{Diff}^{1}\left(\mathbb{R}^{2}\right)$ arbitrarily $C^{1}$-close to $B$ such that $F-B$ is a $\tau$-periodic map and the endomorphism $f$ on $\mathbb{T}^{2}$ induced by $F$ is robustly transitive and has a hyperbolic saddle and a hyperbolic source.

We first deduce the proposition by the claim, postponing its proof. Let $G=$ $Q F Q^{-1}=A+Q(F-B) Q^{-1}$. Since $F-B$ is $\tau$-periodic, $Q(F-B) Q^{-1}$ is 1 periodic. Then $G$ induces a map $g \in E n d^{*}\left(\mathbb{T}^{2}\right)$. Let $\mathcal{U}(g)$ be a small enough neighborhood of $g$. For any $h \in \mathcal{U}(g), h$ can be uniquely lifted to $H \in \operatorname{Diff}^{1}\left(\mathbb{R}^{2}\right)$ satisfying $d(H, G)=d(h, g)$. Since $Q^{-1} H Q=Q^{-1}(H-G) Q+F, Q^{-1} H Q$ induces a map $h^{\prime} C^{1}$-close to $f$. Then $h^{\prime}$ is transitive and $h f_{Q}=f_{Q} h^{\prime}$. Since $f_{Q}$ is a surjection, $h$ is transitive for any $h \in \mathcal{U}(g)$. Since it has a saddle and a source, $h$ is not Anosov.

Proof of the claim. Let $\delta=\frac{1}{k^{5}}, \varepsilon=\frac{1}{k^{100}}, I=[-\delta, \delta], I_{i}=\left[-\frac{\delta+\varepsilon}{k^{i}}+\frac{1}{k^{i}}, \frac{\delta+\varepsilon}{k^{i}}+\frac{1}{k^{i}}\right], 1 \leq$ $i \leq 6$, and $I_{0}=\left[-\frac{\delta+\varepsilon}{k^{6}}, \frac{\delta+\varepsilon}{k^{6}}\right]$. Also, take $t, a, \lambda$ satisfying $0<1-\lambda \ll 1-a \ll t \ll \tau$, which will be precisely stated below.

If $\tau=\frac{1}{k+1}$, by simple calculation we see that $[-\delta,-\delta+\tau) \supset I \cup\left(I_{1}-\tau\right) \cup \bigcup_{i=2}^{5} I_{i}$ $\left(I_{1}-\tau\right.$ denotes the set $\left.\left\{x-\tau \mid x \in I_{1}\right\}\right)$ and $\left(I \cup \bigcup_{i=2}^{5} I_{i}\right) \cap\left(I_{1}-\tau\right)=\emptyset$. For the case $\tau=\frac{1}{k-1}$, we can get a similar property. Then we can take a $\tau$-periodic smooth function $\alpha_{1}$ satisfying that $\alpha_{1}\left(I \cup \bigcup_{i=2}^{5} I_{i}\right)=0$ and $\alpha_{1}\left(I_{1}\right)=t$. Also, take a smooth bump function $\alpha_{2}$ satisfying that $\alpha_{2}\left(\left[-\frac{a \tau}{2}, \frac{a \tau}{2}\right]\right)=1$ and $\alpha_{2}(x)=0$ for any $|x|>\frac{\tau}{2}$. Now construct a map $\Phi: \mathbb{R} \times\left[-\frac{\tau}{2}, \frac{\tau}{2}\right] \rightarrow \mathbb{R}^{2}$ :

$$
\Phi\left(x_{1}, x_{2}\right)=\left(0,\left(\lambda^{\frac{1}{6}}-1\right) \lambda_{2} x_{2} \alpha_{2}\left(x_{2}\right)+\alpha_{1}\left(x_{1}\right)\right) .
$$


It can be regarded as a $\tau$-periodic endomorphism on $\mathbb{R}^{2}$. Let $B_{6}=I_{6} \times S^{1}$. As $t \rightarrow 0$ and $\lambda \rightarrow 1$ (fix $a$ and $\alpha_{2}$ ), $\Phi$ is $C^{1}$ close to 0 and $\left.\Phi\right|_{B_{6}}$ is $C^{1}$ close to the map $\phi: \phi\left(x_{1}, x_{2}\right)=(0, t)$ for fixed $t$ and $a$.

Let $F=\Phi+B, f \in E n d^{*}\left(\mathbb{T}^{2}\right)$ be induced by $F$. Then,

$$
f\left(x_{1}, x_{2}\right)= \begin{cases}\left(\lambda_{1} x_{1}, \lambda^{\frac{1}{6}} \lambda_{2} x_{2}\right), & \left(x_{1}, x_{2}\right) \in\left(I \cup \bigcup_{i=2}^{5} I_{i}\right) \times\left[-\frac{a \tau}{2}, \frac{a \tau}{2}\right], \\ \left(\lambda_{1} x_{1}, \lambda^{\frac{1}{6}} \lambda_{2} x_{2}+t\right), & \left(x_{1}, x_{2}\right) \in I_{1} \times\left[-\frac{a \tau}{2}, \frac{a \tau}{2}\right] .\end{cases}
$$

A $C_{\beta}^{u}$ curve $\gamma \subset[-\delta, \delta] \times S^{1}$ is said to be a $(u, \beta)$-curve of $[-\delta, \delta] \times S^{1}$ if $\partial(\gamma) \subset$ $\partial([-\delta, \delta]) \times S^{1}$. Similarly, we define $(u, \beta)$-curves of $B_{6}$. Now we precisely determine $t, a$ and $\lambda$. Choose $t, a, \lambda$ such that

$$
\frac{\tau}{2}-\lambda \frac{a \tau}{2}<t<\lambda \frac{a \tau}{2}
$$

(i.e, $\left.t<\frac{\tau}{8}\left(<\lambda \frac{a \tau}{2}\right), a>1-\frac{2 t}{\tau}, \lambda>\left(\frac{\tau}{2}-t\right) /\left(\frac{a \tau}{2}\right)\right)$, $a \tau>8 t, f$ has a hyperbolic source in $\{0\} \times\left[0, \frac{\tau}{2}\right]$, and there is a neighborhood $\mathcal{U}_{1}$ of $f$ satisfying the following four conditions:

(1) There exists $\alpha>0$ such that for any $g \in \mathcal{U}_{1}$, any $x \in \mathbb{T}^{2}$ and any unit vector $v \in \mathcal{C}_{\alpha}^{u}(x)$, one has

$$
D g\left(\mathcal{C}_{\alpha}^{u}(x)\right) \subset \mathcal{C}_{\alpha}^{u}(g x), \quad\|D g(v)\|>1,
$$

and for any $(u, \alpha)$-curve $\gamma$ of $B_{6}, g^{6}(\gamma)$ contains a $(u, \alpha)$-curve of $[-\delta, \delta] \times S^{1}$.

(2) For any $g \in \mathcal{U}_{1}$ and any $\left(x_{1}, x_{2}\right) \in B_{6}$,

$$
\frac{t}{2}<g_{2}^{6}\left(x_{1}, x_{2}\right)-x_{2}<2 t
$$

where $g^{6}=\left(g_{1}^{6}, g_{2}^{6}\right)$.

(3) Let $\alpha_{0}=\frac{t}{4}$. For any $g \in \mathcal{U}_{1}$, any $\beta>\frac{\alpha_{0}}{k}$ and any $\mathcal{C}_{k \beta}^{u}$ curve $\gamma \subset B_{6}, g^{6}(\gamma)$ is a $\mathcal{C}_{\beta}^{u}$ curve.

(4) For any $g \in \mathcal{U}_{1}, g$ is not Anosov.

Let $C=I \times\left[-\frac{\tau}{2}, \frac{\tau}{2}\right]$ and $A_{i}=I_{i} \times\left[-\frac{a \tau}{2}, \frac{a \tau}{2}\right], i=0,6$. Note that

$$
f^{6}\left(x_{1}, x_{2}\right)= \begin{cases}\left(k^{6} x_{1}, \lambda x_{2}\right), & \left(x_{1}, x_{2}\right) \in A_{0}, \\ \left(k^{6} x_{1}-1, \lambda x_{2}+t\right), & \left(x_{1}, x_{2}\right) \in A_{6} .\end{cases}
$$

We regard $\left.f^{6}\right|_{A_{0} \cup A_{6}}$ as a map from $A_{0} \cup A_{6}$ to $\left[-\frac{1}{2}, \frac{1}{2}\right]^{2}$. Since $\frac{\tau}{2}-\lambda \frac{a \tau}{2}<t<\lambda \frac{a \tau}{2}$, $\left(C, f^{6}\right)$ is a blender. Let $p_{f}=(0,0)$. Since $W_{l o c}^{u}\left(p_{f}, f\right) \supset[-\delta, \delta] \times\{0\}$, by the properties of a blender, there exists a neighborhood $\mathcal{U}_{2} \subset \mathcal{U}_{1}$ of $f$ such that for any $g \in \mathcal{U}_{2}$

$$
\overline{W^{u}\left(p_{g}, g^{6}\right)} \supset[-\delta, \delta] \times\left[\frac{\tau}{4}, \frac{\tau}{2}\right] \supset[-\delta, \delta] \times\left[\frac{\tau}{4}, \frac{\tau}{4}+2 t\right](\tau>8 t) .
$$

By conditions (1) and (2), we see that for any interval $[a, b]$ satisfying $b-a \geq 2 t$,

$$
g^{6}\left(I_{6} \times[a, b]\right) \supset[-\delta, \delta] \times\left[a+2 t, b+\frac{t}{2}\right] \supset[-\delta, \delta] \times\left[b, b+\frac{t}{2}\right] .
$$

Then $\overline{W^{u}\left(p_{g}, g^{6}\right)} \supset[-\delta, \delta] \times S^{1}$. Note that $f$ uniformly expands over $\mathcal{C}_{\alpha}^{u}$ and preserves $\mathcal{C}_{\alpha}^{u}$ (condition (1)). We deduce that there is a positive integer $m$ such that $g^{m}\left([-\delta, \delta] \times S^{1}\right)=\mathbb{T}^{2}$. Consequently, $\overline{W^{u}\left(p_{g}, g\right)}=\mathbb{T}^{2}$ for any $g \in \mathcal{U}_{2}$.

By condition (1) again, we see that for any $g \in \mathcal{U}_{2}$ and any $\mathcal{C}_{\alpha}^{u}$ curve $\gamma \subset \mathbb{T}^{2}$, there exists a positive integer $m$ such that $g^{m}(\gamma)$ contains a $(u, \alpha)$-curve $\gamma_{1}$ of $B_{6}$. Note that for any $(u, \alpha)$-curve $\gamma^{\prime}$ of $B_{6}, g^{6}\left(\gamma^{\prime}\right)$ contains a $(u, \alpha)$-curve of $[-\delta, \delta] \times S^{1}$ 
and the cone angle of the curve $g^{6}\left(\gamma^{\prime}\right) \cap[-\delta, \delta] \times S^{1}$ uniformly decreases unless $\gamma^{\prime}$ is a $\mathcal{C}_{\alpha_{0}}^{u}$ curve (conditions (1) and (3)). So there is another positive integer $m_{1}$ such that $g^{m_{1}}\left(\gamma_{1}\right)$ contains a $\left(u, \alpha_{0}\right)$-curve $\gamma_{2}$ of $[-\delta, \delta] \times S^{1}$. By conditions $(2)$ and (3), we deduce that every $\mathcal{C}_{\alpha_{0}}^{u}$ curve $\gamma^{\prime}=\left\{\left(x_{1}, \gamma_{2}^{\prime}\left(x_{1}\right)\right) \mid x_{1} \in[-\delta, \delta]\right\}$ contains a $\mathcal{C}_{\alpha_{0}}^{u}$ curve $\gamma^{\prime \prime}=\left\{\left(x_{1}, \gamma_{2}^{\prime \prime}\left(x_{1}\right)\right) \mid x_{1} \in[-\delta, \delta]\right\}$ satisfying that

$$
\begin{gathered}
\min \left\{\gamma_{2}^{\prime \prime}(x)-\gamma_{2}^{\prime}(x) \mid x \in[-\delta, \delta]\right\}>\frac{t}{2}-\alpha_{0}=\frac{t}{4}, \\
\max \left\{\gamma_{2}^{\prime \prime}(x)-\gamma_{2}^{\prime}(x) \mid x \in[-\delta, \delta]\right\}<2 t+\alpha_{0}<3 t .
\end{gathered}
$$

Additionally, $W_{l o c}^{s}\left(p_{f}, f\right) \supset\{0\} \times\left[-\frac{a \tau}{2}, \frac{a \tau}{2}\right]$ and $a \tau>3 t$. Therefore, there exists a neighborhood $\mathcal{U} \subset \mathcal{U}_{2}$ of $f$ such that for any $g \in \mathcal{U}$, there is a positive integer $m_{2}$ satisfying $g^{m_{2}}\left(\gamma_{2}\right) \cap W_{l o c}^{s}\left(p_{g}, g\right) \neq \emptyset$, i.e., $\overline{W^{s}\left(p_{g}, g\right)}=\mathbb{T}^{2}$. By the $\lambda$-lemma, we see that $g$ is transitive for any $g \in \mathcal{U}$.

\section{EXPANDING ENDOMORPHISM}

In this section, we give robustly non-hyperbolic transitive endomorphisms on $\mathbb{T}^{2}$ in all homotopic classes containing expanding maps.

Let $M$ be a smooth Riemannian manifold, $f$ be a regular endomorphism on $M$, and let $X$ be a subset of $M, r>0$, and $B(X, r)=\{x \in M \mid d(x, X)<r\}$. The minimal norm of $D f$ on $X$ is $m\left(\left.D f\right|_{X}\right)=\inf \left\{\frac{\|D f(v)\|}{\|v\|} \mid v \in T_{X} M\right\}$, and $m\left(\left.D f\right|_{M}\right)=$ $m(D f)$. The determinant of $D f$ is $|\operatorname{det}(D f)|=\inf \left\{\left|\operatorname{det}\left(D f_{x}\right)\right| \mid x \in M\right\}$. Let $\delta(f)=\sup \{r \mid \forall x \in M, f$ is diffeomorphic from $B(x, r)$ to its image $\}$.

Let $A \in G L(2, \mathbb{Z})$, whose moduli of eigenvalues are both larger than 1 .

Proposition 4.1. There exists $g \in E n d^{*}\left(\mathbb{T}^{2}\right)$ satisfying that $g$ is homotopic to $f_{A}$ and is robustly non-hyperbolic transitive.

The above proposition is easily deduced by the following two lemmas.

Lemma 4.2. Let $p=(0,0)$. For any $0<\mu<1$ and any neighborhood $U$ of $p$, there exists $g$ homotopic to $f_{A}$ having the following properties:

i) $p$ is a hyperbolic saddle of $g,\left.g\right|_{M \backslash U}=\left.f_{A}\right|_{M \backslash U}$ and $\delta(g)>\frac{\delta\left(f_{A}\right)}{2}$,

ii) $|\operatorname{det}(D g)|>1$ and $m(D g)>\mu$.

Lemma 4.3. Let $f$ be a regular endomorphism on a compact connected Riemannian manifold $M$. Suppose $|\operatorname{det}(D f)|>1, m(D f)=\mu, \delta(f)=7 \delta$, and there is an open subset $U$ of $M$ such that

i) $m\left(\left.D f\right|_{M \backslash U}\right)=\lambda>1$ and $\operatorname{diam}(U)=r<\frac{\lambda-\sqrt{\lambda}}{\lambda} \delta$,

ii) there is an integer $k>0$ satisfying $(\sqrt{\lambda})^{k}>7$ and $\lambda \mu^{k-1}=\lambda_{1}>1$.

Then for any open subset $V$ of $M$, there exists an integer $m>0$ such that $f^{m}(V)=$ $M$. Consequently, $f$ is transitive.

Remark 4.4. Lemma 4.2 gives a specially derived Anosov map. Both Lemma 4.2 and Proposition 4.1 are valid for any expanding endomorphism on $\mathbb{T}^{n}$. In Sh1, Shub proved that "any expanding endomorphism on any compact manifold is transitive," and the non-trivial fact that "any expanding endomorphism on $\mathbb{T}^{n}$ is homotopic to and topologically conjugate to a linear expanding endomorphisms."

To show Lemma 4.3, we need the following simple lemma. 
Lemma 4.5. Let $f$ be a regular endomorphism on a compact Riemannian manifold M. Then:

(i) Suppose $V$ is an open subset of $M$ and $m\left(\left.D f\right|_{V}\right)=\mu$. Then for any $B(x, \tau)$ $\subset V, f(B(x, \tau)) \supset B(f x, \mu \tau)$.

(ii) Suppose there is an open subset $U$ of $M$ such that $m\left(\left.D f\right|_{M \backslash U}\right)=\mu>\mu^{\prime}$ and $\operatorname{diam}(U)=r$. Then for any $\tau>\frac{\mu}{\mu-\mu^{\prime}} r, f(B(x, \tau)) \supset B\left(f x, \mu^{\prime} \tau\right)$. Also, for any $x, y \in M$ satisfying $\frac{\mu}{\mu-\mu^{\prime}} r<d(x, y)<\delta(f), d(f x, f y)>\mu^{\prime} d(x, y)$.

Proof. The proof of (i) is quite easy. We only prove (ii). For any smooth curve $\gamma:[a, b] \rightarrow M$, let $t_{1}=\inf \{t \mid \gamma(t) \in U\}$ and $t_{2}=\sup \{t \mid \gamma(t) \in U\}$. Then $d\left(\gamma\left(t_{1}\right), \gamma\left(t_{2}\right)\right) \leq r$ and $\ell(f \gamma)=\int_{a}^{b}|D f(\dot{\gamma}(t))| d t \geq \mu\left(\int_{a}^{t_{1}}|\dot{\gamma}(t)| d t+\int_{t_{2}}^{b}|\dot{\gamma}(t)| d t\right)$. So

$$
\operatorname{diam}(\gamma) \leq \int_{a}^{t_{1}}|\dot{\gamma}(t)| d t+\int_{t_{2}}^{b}|\dot{\gamma}(t)| d t+r \leq r+\frac{\ell(f \gamma)}{\mu} .
$$

For any $x, z \in M$, there exists a shortest geodesic $\gamma$ from $f x$ to $z$. Since $f$ is a local diffeomorphism and a covering map ( $M$ is compact), there is a smooth lift $\gamma_{1}$ of $\gamma$, i.e., a smooth curve $\gamma_{1}$ from $x$ to $w$ satisfying $f \gamma_{1}=\gamma$. If $z \in B\left(f x, \mu^{\prime} \tau\right)$, then $d(x, w) \leq \operatorname{diam}\left(\gamma_{1}\right) \leq r+\frac{\mu^{\prime} \tau}{\mu}<\tau$, i.e., $f(B(x, \tau)) \supset B\left(f x, \mu^{\prime} \tau\right)$. If $z=f y$ and $\frac{\mu}{\mu-\mu^{\prime}} r<d(x, y)<\delta(f)$, then $d(x, w) \leq r+\frac{d(f x, f y)}{\mu}$. Note that $f(w)=f(y)$ and $d(x, y)<\delta(f)$. Then, by the definition of $\delta(f)$, we see that $d(x, w) \geq d(x, y)$. So $d(f x, f y)>\mu^{\prime} d(x, y)$.

Proof of Lemma 4.3. Since $|\operatorname{det}(D f)|>1$, for any connected open set $V$ of $M$, there exists a positive integer $n_{0}$ such that $\operatorname{diam}\left(f^{n_{0}}(V)\right) \geq \delta(f)=7 \delta$ (otherwise, $\operatorname{Volume}\left(f^{n}(V)\right) \rightarrow \infty$ as $\left.n \rightarrow \infty\right)$. Then we can take a (continuous) curve $\gamma_{0} \subset f^{n_{0}}(V)$ such that $\operatorname{diam}\left(\gamma_{0}\right)=\delta, B\left(\gamma_{0}, \varepsilon\right) \subset f^{n_{0}}(V)$ and $B\left(\gamma_{0}, \delta\right) \cap U=\emptyset$. Note that $B\left(\gamma_{0}, \delta\right) \cap U=\emptyset$ and $m\left(\left.D f\right|_{M \backslash U}\right)=\lambda$. Then, by i) of the above lemma, $f\left(B\left(\gamma_{0}, \varepsilon\right)\right) \supset B\left(f\left(\gamma_{0}\right), \lambda \varepsilon\right)$. Since $m(D f)>\mu$, by i) of the above lemma again, we see that $f^{k}\left(B\left(\gamma_{0}, \varepsilon\right)\right) \supset f^{k-1}\left(B\left(f\left(\gamma_{0}\right), \lambda \varepsilon\right)\right) \supset B\left(f^{k}\left(\gamma_{0}\right), \lambda \mu^{k-1} \varepsilon\right)=B\left(f^{k}\left(\gamma_{0}\right), \lambda_{1} \varepsilon\right)$. Also, note that $\delta(f)=7 \delta>\delta>\frac{\lambda}{\lambda-\sqrt{\lambda}} r$ and $m\left(\left.D f\right|_{M \backslash U}\right)>\lambda$. So, by the latter part of (ii) of the above lemma $\left(\mu=\lambda>\sqrt{\lambda}=\mu^{\prime}\right), \operatorname{diam}\left(f^{k}\left(\gamma_{0}\right)\right)>$ $\min \left\{(\sqrt{\lambda})^{k} \delta, \delta(f)\right\}=7 \delta$. Then, the connected set $f^{k}\left(\gamma_{0}\right)$ contains a curve $\gamma_{1}$ such that $\operatorname{diam}\left(\gamma_{1}\right)=\delta$ and $B\left(\gamma_{1}, \delta\right) \cap U=\emptyset$. Take a positive integer $l$ satisfying $\delta<\lambda_{1}{ }^{l} \varepsilon \leq \lambda_{1} \delta$. Inductively, we can find curves $\left\{\gamma_{n}\right\}_{0 \leq n \leq l}$ such that

$\operatorname{diam}\left(\gamma_{n}\right)=\delta, B\left(\gamma_{n}, \delta\right) \cap U=\emptyset$, and $f^{k}\left(B\left(\gamma_{n}, \lambda_{1}{ }^{n} \varepsilon\right)\right) \supset B\left(\gamma_{n+1}, \lambda_{1}{ }^{n+1} \varepsilon\right), 0 \leq n<l$. Then $f^{\left(n_{0}+k l\right)}(V) \supset B\left(\gamma_{l}, \delta\right)$. Since $\delta>\frac{\lambda}{\lambda-\sqrt{\lambda}} r, f^{\left(2 j+n_{0}+l k\right)}(V)$ contains $B\left(f^{2 j}\left(\gamma_{l}\right)\right.$, $\lambda^{j} \delta$ ) for any positive integer $j$. Thus, there is a positive integer $m$ such that $f^{m}(V)=M$.

The proof of Lemma 4.2 is quite basic. For completion, we give its proof.

Proof of Lemma 4.2. Take a basis $\left\{e_{1}, e_{2}\right\}$ of $\mathbb{R}^{2}$ such that the representation of $A$ under the basis is the following: $\left(\begin{array}{cc}\lambda_{1} & 0 \\ 0 & \lambda_{2}\end{array}\right),\left|\lambda_{1}\right| \geq\left|\lambda_{2}\right|>1$, or $\left(\begin{array}{ll}\lambda & 0 \\ \alpha & \lambda\end{array}\right),|\lambda|>$ $|\alpha|+1$, or $\lambda R_{\theta}=\lambda\left(\begin{array}{ll}\cos \theta & \sin \theta \\ -\sin \theta & \cos \theta\end{array}\right),|\lambda|>1$. Take the norm of $\mathbb{R}^{2}$ such that 
$\left\{e_{1}, e_{2}\right\}$ is an orthonormal basis which induces the flat Riemannian metric on $\mathbb{T}^{2}$. Here we use this Riemannian metric on $\mathbb{T}^{2}$.

Fix a small neighborhood $U$ of $p$. Construct $f \in E n d^{*}\left(\mathbb{T}^{2}\right)$ in the homotopic class of $f_{A}$ such that $\left.f\right|_{M \backslash U}=\left.f_{A}\right|_{M \backslash U},\left.f\right|_{V}\left(x_{1}, x_{2}\right)=\left(\lambda_{1} x_{1}, \lambda_{2} x_{2}\right), V \subset U$ is a neighborhood of $p,\left|\lambda_{1}\right| \geq\left|\lambda_{2}\right|>1$, and $m(D f)>1$. Then $|\operatorname{det}(D f)| \geq m(D f)^{2}>$ 1. The construction is not difficult for the first two cases of $A$. Suppose $A=$ $\lambda R_{\theta}$. It is trivial to show that there is a large enough positive integer $m, \alpha=\frac{\theta}{m}$, $\left\{\varepsilon_{i}\right\}_{i=1}^{\infty}, \varepsilon_{i} \searrow 0$, and the smooth diffeomorphisms $\left\{\Phi_{i}\right\}_{i=1}^{\infty}$ on $\mathbb{R}^{2}$ such that, for any positive integer $i$,

$$
m\left(D \Phi_{i}\right)>\frac{1}{\lambda} ; \Phi_{i}(x)=R_{-\alpha}(x),|x|<\varepsilon_{i+1} ; \Phi_{i}(x)=x,|x|>\varepsilon_{i} .
$$

Suppose $B\left(p, \varepsilon_{1}\right) \subset U$. Let $f=\lambda \Phi_{m} \ldots \Phi_{1} R_{\theta}$. Note that $f(x)=\lambda \Phi_{i} R_{(\theta-(i-1) \alpha)}(x)$ for $|x| \in\left[\varepsilon_{i+1}, \varepsilon_{i}\right]$. We can see that $m(D f)>1$ and $f(x)=\lambda x$ for any $|x|<\varepsilon_{m+1}$. Take any $v, \varepsilon>0$ satisfying $\left|v \lambda_{1}\right|>1$ and $v+\varepsilon<1$. Suppose $V \supset I_{1} \times I_{2}, I_{1}$ and $I_{2}$ are two open intervals whose central points are both 0 , and $\frac{3\left|\lambda_{2}\right| \ell\left(I_{2}\right)}{\ell\left(I_{1}\right)}<\varepsilon$. Let $\alpha_{1}$ be a smooth bump function such that $\alpha_{1}(x)=1$ for any $x$ contained in a small neighborhood of $0, \alpha_{1}(x)=0$ for any $x \notin I_{1}, 0 \leq \alpha_{1}(x) \leq 1$ and $\left|\alpha_{1}{ }^{\prime}(x)\right|<\frac{3}{\ell\left(I_{1}\right)}$. Take another smooth function $\alpha_{2}$ such that $\alpha_{2}(0)=0,\left|\alpha_{2}{ }^{\prime}(0)\right|=v+\varepsilon, \alpha_{2}(x)=\lambda_{2} x$ for any $x \notin I_{2}$, and $\lambda_{2} \alpha_{2}{ }^{\prime}(x)>\left|\lambda_{2}\right|(v+\varepsilon)$. Take $g$ in the homotopic class of $f$ :

$$
g\left(x_{1}, x_{2}\right)=\left(\lambda_{1} x_{1}, \alpha_{2}\left(x_{2}\right) \alpha_{1}\left(x_{1}\right)+\lambda_{2} x_{2}\left(1-\alpha_{1}\left(x_{1}\right)\right) .\right.
$$

We can see that $g$ has the hyperbolic saddle $p$ and $\delta(g)>\frac{\delta\left(f_{A}\right)}{2}$. Also, for any $\left(x_{1}, x_{2}\right) \in U$,

$$
D g_{\left(x_{1}, x_{2}\right)}=\left(\begin{array}{ll}
\lambda_{1} & 0 \\
\alpha_{1}{ }^{\prime}\left(x_{1}\right)\left(\alpha_{2}\left(x_{2}\right)-\lambda_{2} x_{2}\right) & \alpha_{2}{ }^{\prime}\left(x_{2}\right) \alpha_{1}\left(x_{1}\right)+\lambda_{2}\left(1-\alpha_{1}\left(x_{1}\right)\right)
\end{array}\right) .
$$

Note that $\left|\alpha_{2}{ }^{\prime}\left(x_{2}\right) \alpha_{1}\left(x_{1}\right)+\lambda_{2}\left(1-\alpha_{1}\left(x_{1}\right)\right)\right|>v+\varepsilon$ and $\left|\alpha_{1}^{\prime}\left(x_{1}\right)\left(\alpha_{2}\left(x_{2}\right)-\lambda_{2} x_{2}\right)\right|<$ $\frac{3}{\ell\left(I_{1}\right)} \times\left|\lambda_{2}\right| \ell\left(I_{2}\right)<\varepsilon$. Then for any $x \in U,\left|\operatorname{det}\left(D g_{x}\right)\right|>\left|\lambda_{1} v\right|>1$. Also note that for any matrix $B=\left(\begin{array}{ll}a & 0 \\ b & c\end{array}\right), m(B) \geq \min \{|a|,|c|\}-|b|$. So $m(D g)>v$.

Proof of Theorem 1.1. Now, Theorem 1.1 follows from Proposition 3.7 and Proposition 4.1.

\section{ACKNOWLEDGMENTS}

The authors would like to thank Yi Shi, Fan Yang, Xuan Zhang, Lan Wen, Dawei Yang, Xiao Wen, and Ming Li for discussions on blenders.

\section{REFERENCES}

[ABCD] F. Abdenur, C. Bonatti, S. Crovisier and L. J. Díaz, Generic diffeomorphisms on compact surfaces, Fund. Math., 187 (2005), 127-159. MR2214876 (2006m:37024)

[ABD] F. Abdenur, C. Bonatti and L. J. Díaz, Non-wandering sets with non-empty interiors, Nonlinearity, 17 (2004), 175-191. MR2023438(2004m:37031)

[AH] N. Aoki and K. Hiraide, Topological Theory of Dynamical Systems, North-Holland, 1994. MR.1289410 (95m:58095)

[AP] R. Adler and R. Palais, Homeomorphic conjugacy of automorphisms on the torus, Proc. Amer. Math. Soc., 16 (1965), 1222-1225. MR0193181 (33:1402)

[AY] J. C. Alexander and J. A. Yorke, Fat baker's transformations, Ergodic Theory Dynam. Systems, 4 (1984), 1-23. MR758890 (86c:58090) 
[Be] P. Berger, Persistence of stratifications of normally expanded laminations, C. R. Math. Acad. Sci. Paris, 346 (2008), 767-772. MR2427079 (2010c:37058)

[BD] C. Bonatti and L. J. Díaz, Persistent nonhyperbolic transitive diffeomorphisms, Ann. of Math., 143 (1996), 357-396. MR1381990(97d:58122)

[BDP] C. Bonatti, L. J. Díaz and E. Pujals, A $C^{1}$-dichotomy for diffeomorphisms: Weak forms of hyperbolicity or infinitely many sinks or sources, Ann. of Math, 158 (2003), 355-418. MR2018925 (2007k:37032)

[BKRU] R. Bamón, J. Kiwi, J. Rivera-Letelier and R. Urzúa, On the topology of solenoidal attractors of the cylinder, Ann. Inst. H. Poincaré Anal. Non Linéaire, 23 (2006), 209236. MR 2201152 (2007b:37042)

[D] L. J. Díaz, Cycles, partial hyperbolicity, and transitivity, http://www.mat.puc-rio.br/ lodiaz/publ.html.

[DPU] L. J. Díaz, E. Pujals and R. Ures, Partial hyperbolicity and robust transitivity, Acta Math., 183 (1999), 1-43. MR1719547(2002a:37039)

[HHU] F. Rodiguez Hertz, M. Rodriguez Hertz and R. Ures, A survey of partially hyperbolic dynamics, in "Partially Hyperbolic Dynamics, Laminations, and Teichmüller Flow", Fields Inst. Comm., 51, AMS, 2007, 35-87. MR2388690 (2009f:37032)

[L] P. Liu, Stability of orbit spaces of endomorphisms, Manuscripta Math., 93 (1997), 109128. MR $1446193(98 \mathrm{e}: 58107)$

[LP] C. Lizana and E. Pujals, Robust transitivity for endomorphisms, Ergodic Theory and Dynam. Systems, to appear, http://dx.doi.org/10.1017/s0143385712000247

[M1] R. Mané, Contributions to the $C^{1}$-stability conjecture, Topology, 17 (1978), 386-396. MR.516217 (84b:58061)

[M2] R. Mané, An ergodic closing lemma, Ann. of Math., 116 (1982), 503-540. MR678479 (84f:58070)

[Mo] K. Moriyasu, The ergodic closing lemma for $C^{1}$ regular maps. Tokyo J. Math., 15 (1992), 171-183. MR 1164194 (93e:58111)

[P] F. Przytycki, Anosov endomorphisms, Studia Math., 58 (1976), 249-285. MR0445555 $(56: 3893)$

[Sh1] M. Shub, Endomorphisms of compact differentiable manifolds, Amer. J. Math., 91 (1969), 175-199. MR0240824 (39:2169)

[Sh2] M. Shub, Topologically transitive diffeomorphisms on $\mathbb{T}^{4}$, Lect. Notes in Math., 206, Springer, 1971, 39.

[Su] N. Sumi, A class of differentiable toral maps which are topologically mixing, Proc. Amer. Math. Soc., 127 (1999), 915-924. MR1469436 (99f:58144)

[T] M. Tsujii, Fat solenoidal attractors, Nonlinearity, 14 (2001), 1011-1027. MR.1862809 (2002j:37035)

[W] R. Williams, The "DA" maps of Smale and structural stability, in "Global Analysis", Proc. Sympos. Pure Math., XIV, AMS, 1970, 329-334. MR0264705 (41:9296)

School of Mathematical Sciences, Peking University, Beijing 100871, People's RePUBLIC OF CHINA

E-mail address: hebaolin@pku.edu.cn

School of Mathematical Sciences, Peking University, Beijing 100871, People's RePUBLIC OF CHINA

E-mail address: gansb@math.pku.edu.cn 